SCIEnTIA Marina 70 (3)

September 2006, 389-398, Barcelona (Spain)

ISSN: 0214-8358

\title{
Marine ornamental species from European waters: a valuable overlooked resource or a future threat for the conservation of marine ecosystems?
}

\author{
RICARDO CALADO \\ CCMAR, Universidade do Algarve, Campus de Gambelas, 8000-117 Faro, Portugal \\ Templo Aquático, Rua de Santa Marta 27K, 1150-291 Lisboa, Portugal. E-mail: rjcalado@ hotmail.com
}

\begin{abstract}
SUMMARY: The worldwide growth of the marine aquarium market has contributed to the degradation of coral reef ecosystems. Enforcing the legislation on importing ornamental species has led some European traders to concentrate on local species. Portugal is used as a case study of marine ornamental fish and invertebrate collection in European waters. One hundred and seventy two species occurring in Portuguese waters (mainland, the Azores and Madeira archipelagos) were considered as potential targets for the marine aquarium industry, some of which are already traded on a regular basis (e.g. Clibanarius erythropus, Lysmata seticaudata, Cerithium vulgatum, Hinia reticulata and Ophioderma longicauda). To ensure appropriate management and conservation of these resources, the following options have been evaluated: banning the harvest and trade of all marine ornamental species from European waters; creating sanctuaries and "no take zones"; issuing collection permits; creating certified wholesalers; implementing the use of suitable gear and collecting methods; setting minimum and maximum size limits; establishing species-based quotas; protecting rare, or "key stone" species and organisms with poor survivability in captivity; establishing closed seasons; culturing ornamental organisms; and creating an "eco-fee" to support research and management. Establishing this sustainable alternative fishery may help minimise the economical and social impacts caused by the crash of important food fisheries in Portugal and other European and West African countries.
\end{abstract}

Keywords: marine ornamentals, aquarium trade, marine conservation, marine resources, management, alternative fisheries.

RESUMEN: ESPECIES MARINAS ORNAMENTALES DE AGUAS EUROPEAS: ¿UN RECURSO VALIOSO PASADO POR ALTO O UNA AMENAZA FUTURA PARA LA CONSERVACIÓN DE ECOSISTEMAS MARINOS? - El crecimiento a nivel mundial del comercio para acuarios marinos ha contribuido a la degradación de ecosistemas de arrecifes de coral. La aplicación de la legislación referente a importaciones de especies ornamentales ha inducido a algunos comerciantes europeos a concentrarse en especies locales. Portugal se utiliza como caso específico para la recolección de peces e invertebrados marinos en aguas europeas. Un total de 172 especies presentes en aguas portuguesas (península, Azores y Madeira) se consideran objetivos potenciales para la industria de acuarios marinos, siendo algunas de ellas ya el objeto de un comercio regular (p.e. Clibanarius erythropus, Lysmata seticaudata, Cerithium vulgatum, Hinia reticulata y Ophioderma longicauda). A fin de asegurar la correcta gestión y conservación de estos recursos, las siguientes opciones fueron evaluadas: prohibición de la recolección y comercio de todas las especies marinas ornamentales de aguas europeas; creación de santuarios y zonas de veda; emisión de permisos de recolección; provisión de certificados acreditados a mayoristas; implementación del uso de artes y métodos de recolección adecuados; establecimiento de límites de tallas máximas y mínimas; establecimiento de cuotas para cada especie; protección de especies raras, especies claves y de organismos con débil supervivencia en cautividad; establecimiento de vedas estacionales; cultivo de organismos ornamentales; y creación de una eco-tasa para potenciar la investigación y la gestión. El establecimiento de esta alternativa pesquera sostenible puede ayudar a minimizar el impacto social y económico causado por el colapso de importantes pesquerías en Portugal y otros países europeos y de África occidental.

Palabras clave: especies marinas ornamentales, conservación marina, recursos marinos, gestión, pesquerías alternativas. 


\section{INTRODUCTION}

The increase in coastal development in reef areas, the practice of coral dredging for construction purposes, dynamite fishing, global environmental changes and other impacts have led coral reefs to crisis state (Bryant et al., 1998). These complex and fragile ecosystems have been the main suppliers of tropical marine ornamentals for a long time (Wood, 1985; Wabnitz et al., 2003). Unfortunately, the worldwide growth in the trade of these highly priced species for the aquarium market has also contributed to the degradation of these habitats. As usually happens when any natural resource is exploited, countries involved in this billion-dollar industry are now facing several problems (Olivier, 2003). Some of the most concerning are the over-harvesting of ornamental species, the ecological changes induced by ornamental collection, and a general degradation of reefs due to the use of unsuitable collection gears and poisoning by cyanide (Wood, 2001; Wabnitz et al., 2003). The increasing global awareness of the negative impacts of collecting ornamental species has led collectors, traders, researchers and hobbyists to evaluate sustainable solutions for these important fisheries. Certainly, one of the solutions would be to ban the collection of ornamental species completely. However, collecting these species is an important income source for most exporting countries (e.g. Daw et al., 2001), and the economical and social impacts of complete banning would certainly be disastrous. Nevertheless, creating protective reserve areas where ornamental collection is prohibited has proven to be a highly effective strategy for managing these resources (Friedlander, 2001). Using acceptable collecting methods and gears has also ensured minimal damage both to stock and habitat and reduced post-harvest losses. In addition to these measures, establishing size limits for certain species and species-based quotas, protecting "key-stone" species, introducing closed seasons and limiting the number of collectors have also proven to be appropriate management strategies (Wood, 2001). An alternative approach to using wild reef organisms is the aquaculture of ornamental species. However, with only about 5 to $10 \%$ of the marine species available in the aquarium market produced in captivity, this approach is still far from ensuring that the increasing demand for marine ornamental organisms can be exclusively fulfilled with specimens raised in captivity (Chapman et al., 1997; Tlusty, 2002).
An important step for protecting reef habitats is tighter control of importation of ornamental species by some of the major importing countries - the USA and EU countries. Although in EU countries this control has been mainly focused on CITES listed species (namely stony corals and giant clams) (Bruckner, 2001), the increased enforcement of the legislation on importing ornamental species has led some traders to reconsider their market policies. In Portugal, the main consequence of this law enforcement has been an attempt by marine ornamental traders to concentrate on local species. It is already common to find the hermit crab Clibanarius erythropus (Latreille, 1818), the Monaco shrimp Lysmata seticaudata (Risso, 1816), the cerith snail Cerithium vulgatum Bruguiere, 1789, the nassa snail Hinia reticulata (Linnaeus, 1758) and the serpent star Ophioderma longicauda (Retzius, 1805) for sale in Portuguese aquarium stores.

The main criteria marine species from warm temperate and subtropical waters should fulfil to achieve "ornamental" status is the ability to tolerate tropical aquarium temperatures (ranging from 26 to $30^{\circ} \mathrm{C}$ ), be "hardy", display a striking coloration and be "reef-safe" (not harming other inhabitants of the reef aquarium) (Calado et al., 2003a). However, if a certain species only lacks the coloration requirement but displays a unique appearance (such as mimetic species), or performs a specific function in the reef aquarium (such as eating algae or "nuisance organisms") it may also be reasonably targeted by the ornamental industry.

Despite the existence of strict European legislation on food fisheries, the capture and trade of ornamental species in European waters has never been addressed. This lack of legislation, associated with the high market prices marine ornamental species can attain, may lead to unsustainable use of these new and valuable resources, further impairing the conservation of marine habitats.

The objective of this work is to use the Portuguese situation as a case study, by presenting a list of invertebrate and fish species occurring in Portuguese waters that may be potential target species for the marine ornamental industry and suggesting management and conservation measures.

\section{MATERIALS AND METHODS}

The members of genera or families of the most heavily traded invertebrate groups and fishes 
described by Fosså and Nielsen (2000), Michael (2001) and Sprung (2001) occurring in Portuguese waters (Portugal mainland, the Azores and Madeira archipelagos) were evaluated as potential target species for the marine aquarium trade industry. The majority of organisms unable to stand the temperatures of tropical marine aquariums (Calado, unpublished data) were excluded from this preliminary list. However, some of the organisms unable to tolerate the warmer temperatures of reef aquariums, but superbly coloured and appealing were included in the present list. To evaluate the potential economic profitability of marine ornamental collection in Portuguese waters, average commercial values are presented based on year round surveys of the main Portuguese aquarium retail stores. Highly priced species and species inhabiting the intertidal region, where large numbers of specimens can be easily collected, were considered to be more vulnerable to the marine ornamental trade, and the ones that should have their collection and trade most readily regulated.

\section{RESULTS}

One hundred and seventy two species occurring in Portuguese waters were considered as potential target species for the marine ornamental trade industry (Fig. 1). The 109 invertebrate species are listed in Tables 1, 2 and 3 and are mainly represented by decapod crustaceans (32 species) and molluscs (29 species). The 63 marine fish species are listed in Table 4 and are mainly represented by the families

TABLE 1. - Potential marine ornamental sponge, cnidarians and segmented worm species from Portuguese waters (A, Azores; M, Madeira; P, Portugal mainland).

\begin{tabular}{|c|c|c|c|}
\hline Families & Species & Common name & Occurrence \\
\hline \multicolumn{4}{|l|}{ Sponges } \\
\hline Aplisinidae & Aplysina aerophoba & Golden sponge & $\mathrm{M}, \mathrm{P}$ \\
\hline \multirow[t]{4}{*}{ Axinelidae } & Acanthella acuta & Spiny sponge & $\mathrm{P}$ \\
\hline & Axinella polypoides & Finger sponge & $\mathrm{P}$ \\
\hline & Axinella verrucosa & Finger sponge & $\mathrm{P}$ \\
\hline & Haliclona oculata & Tube sponge & $\mathrm{P}$ \\
\hline \multirow[t]{2}{*}{ Clathrinidae } & Clathrina clathrus & White clathrina & $\mathrm{P}$ \\
\hline & Clathrina coriacea & Yellow clathrina & M \\
\hline Hymeniacidonidae & Hemimycale columella & Crater sponge & $\mathrm{M}, \mathrm{P}$ \\
\hline \multirow{2}{*}{ Irciniidae } & Ircinia fasciculata & Stinking sponge & $\mathrm{P}$ \\
\hline & Ircinia muscarum & Dark stinking sponge & $\mathrm{P}$ \\
\hline Spongiidae & Spongia agaricina & Elephant ear & $\mathrm{P}$ \\
\hline \multicolumn{4}{|l|}{ Cnidarians } \\
\hline Actiniidae & Anemonia sulcata & Snakelocks anemone & $\mathrm{M}, \mathrm{P}$ \\
\hline Aiptasiidae & Aiptasia mutabilis & Trumpet anemone & $\mathrm{M}, \mathrm{P}$ \\
\hline \multirow[t]{4}{*}{ Alcyonidae } & Alcyonium acaule & Broccoli coral & $\mathrm{P}$ \\
\hline & Alcyonium coralloides & Broccoli coral & $\mathrm{P}$ \\
\hline & Alcyonium glomeratum & Broccoli coral & $\mathrm{P}$ \\
\hline & Alcyonium palmatum & Broccoli coral & M \\
\hline Anthipatidae & Antipathes subpinnata & Black coral & $\mathrm{A}, \mathrm{M}, \mathrm{P}$ \\
\hline Cerianthidae & Cerianthus membranaceus & Tube dwelling anemone & M, P \\
\hline Corallimorphidae & Corynactis viridis & Jewel anemone & A, M, P \\
\hline \multirow[t]{2}{*}{ Dendrophylliidae } & Dendrophyllia ramea & Tree coral & M \\
\hline & Leptosammia pruvoti & Yellow solitary coral & $\mathrm{P}$ \\
\hline Gerardiidae & Gerardia savaglia & Encrusting anemone & M \\
\hline \multirow[t]{3}{*}{ Gorgoniidae } & Leptogorgia ruberrima & Red sea fan & $\mathrm{P}$ \\
\hline & Lophogorgia sarmentosa & Yellow sea fan & M \\
\hline & Lophogorgia vimnalis & Red sea fan & M \\
\hline Isophelliidae & Telmatactis cricoides & Club tipped anemone & $\mathrm{M}$ \\
\hline Parazoanthidae & Parazoanthus axinellae & Sponge zoanthid & $\mathrm{P}$ \\
\hline Sagartidae & Actinothoe sphyrodeta & Daisy anemone & $\mathrm{M}, \mathrm{P}$ \\
\hline Zoanthidae & Palythoa canariense & Canarian sea mat & M \\
\hline \multicolumn{4}{|l|}{ Segmented worms } \\
\hline \multirow[t]{3}{*}{ Sabbelidae } & Sabella pavonina & Spiral feather duster & $\mathrm{A}, \mathrm{M}, \mathrm{P}$ \\
\hline & Sabella spallanzanii & Peacock feather duster & A, M, P \\
\hline & Bispira volutacornis & Cluster feather duster & M \\
\hline \multirow[t]{3}{*}{ Serpulidae } & Filograna sp. & Hard tube duster & $\mathrm{P}$ \\
\hline & Protula tubularia & Hard tube cluster duster & $\mathrm{P}$ \\
\hline & Serpula vermicularis & Variable tube worm & $\mathrm{A}, \mathrm{M}, \mathrm{P}$ \\
\hline Terebelidae & Eupolymnia nebulosa & Spaghetti worm & M, P \\
\hline
\end{tabular}




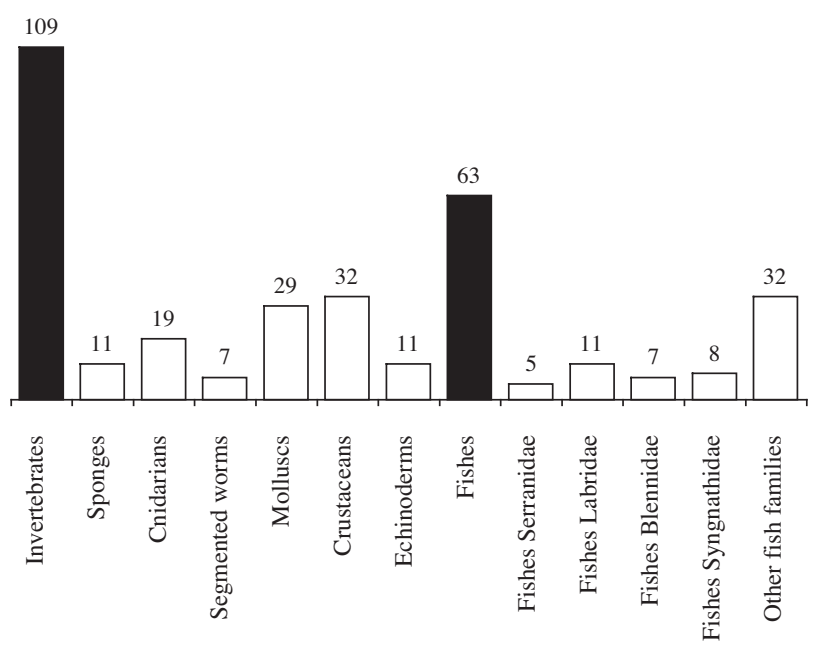

FIG. 1 - Potential marine ornamental invertebrate and fish species from Portuguese waters (Azores, Madeira and Portugal mainland).

Labridae (11 species), Syngnathidae (8 species), Blennidae (7 species) and Serranidae (5 species). Certain species, such as the members of the families Nassariidae, Cerithiidae, Columbellidae, Fissurelidae, the crustaceans Percnon gibbesi, Clibanarius erythropus and C. aequabilis, the serpent star Ophioderma longicauda and the blennies Coryphoblenius galerita and Parablennius parvi- cornis, are also listed in Tables 1, 2, 3 and 4 not due to any attractive feature, but rather because they are popular members of "reef aquarium cleaning crews" (see Sprung, 2001), responsible for eating algae or "nuisance organisms" or scavenging on detritus. The estimated commercial values of selected highly priced ornamental invertebrates and fish are listed in Table 5 and 6 respectively. The species labelled as "cleaning crew" members are also listed in Tables 5 and 6 because they are generally purchased in considerable numbers by the majority of hobbyists. The invertebrate and fish species considered as most vulnerable to the ornamental trade are also summarised in Table 5 and 6 respectively. Again, "cleaning crew" members were listed because of their popularity and due to the fact that they can be easily captured in considerable numbers, either by traders or hobbyists, in intertidal regions or shallow water that is accessible with snorkelling gear.

\section{DISCUSSION}

The large number of potential target species for the marine ornamental industry occurring in

TABLE 2. - Potential marine ornamental mollusc species from Portuguese waters (A, Azores; M, Madeira; P, Portugal mainland). * See text for explanation.

\begin{tabular}{|c|c|c|c|}
\hline Families & Species & Common name & Occurrence \\
\hline \multicolumn{4}{|l|}{ Molluscs } \\
\hline \multirow[t]{2}{*}{ Cerithiidae } & Cerithium rupestre* & Common cerith & $\mathrm{P}$ \\
\hline & Cerithium vulgatum* & Cerith & $\mathrm{M}, \mathrm{P}$ \\
\hline \multirow[t]{3}{*}{ Columbellidae } & Columbella rustica* & Dove shell & $\mathrm{A}, \mathrm{M}, \mathrm{P}$ \\
\hline & Mitrella gervillei* & Dove shell & $\mathrm{P}$ \\
\hline & Mitrella scripta* & Dove shell & M, P \\
\hline \multirow[t]{4}{*}{ Cypraeidae } & Erosaria spurca & Cowries & $\mathrm{A}, \mathrm{M}, \mathrm{P}$ \\
\hline & Luria lurida & Cowries & M, P \\
\hline & Schilderia achatidea & Cowries & $\mathrm{M}, \mathrm{P}$ \\
\hline & Zonaria pyrum & Cowries & $\mathrm{P}$ \\
\hline \multirow[t]{3}{*}{ Fissurelidae } & Diodora gibberula* & Keyhole limpet & $\mathrm{P}$ \\
\hline & Diodora graeca* & Keyhole limpet & $\mathrm{M}, \mathrm{P}$ \\
\hline & Diodora italica* & Keyhole limpet & $\mathrm{P}$ \\
\hline \multirow[t]{3}{*}{ Limidae } & Lima exilis & File shell & A, P \\
\hline & Lima hians & Hian's file shell & $\mathrm{A}, \mathrm{M}, \mathrm{P}$ \\
\hline & Lima lima & Greater file shell & M, P \\
\hline \multirow[t]{6}{*}{ Nassariidae } & Hima incrassatus* & Nassa snail & $\mathrm{P}$ \\
\hline & Hinia reticulata* & Nassa snail & $\mathrm{M}, \mathrm{P}$ \\
\hline & Nassarius elatus* & Nassa snail & $\mathrm{P}$ \\
\hline & Nassarius nitidus* & Nassa snail & $\mathrm{M}, \mathrm{P}$ \\
\hline & Niotha denticulatus* & Nassa snail & $\mathrm{M}, \mathrm{P}$ \\
\hline & Uzita lima* & Nassa snail & M, P \\
\hline Octopodidae & Octopus macropus & White spotted octopus & $\mathrm{A}, \mathrm{M}, \mathrm{P}$ \\
\hline Patellidae & Helcion pellucidum & Translucid limpet & $\mathrm{P}$ \\
\hline \multirow{3}{*}{ Pinnidae } & Atrina pectinata & Smaller pen shell & $\mathrm{P}$ \\
\hline & Pinna nobilis & Greater pen shell & $\mathrm{P}$ \\
\hline & Pinna rudis & Pen shell & A, M \\
\hline Spondylidae & Spondylus gaederopus & Thorny oyster & $\mathrm{A}, \mathrm{M}, \mathrm{P}$ \\
\hline Turbinidae & Bolma rugosa* & Star snail & $\mathrm{A}, \mathrm{M}, \mathrm{P}$ \\
\hline Vermetidae & Serpulorbis arenaria & Worm shell & $\mathrm{P}$ \\
\hline
\end{tabular}


TABlE 3. - Potential marine ornamental crustacean and echinoderm species from Portuguese waters (A, Azores; M, Madeira; P, Portugal mainland). * See text for explanation.

\begin{tabular}{|c|c|c|c|}
\hline Families & Species & Common name & Occurrence \\
\hline \multicolumn{4}{|l|}{ Crustaceans } \\
\hline \multirow[t]{2}{*}{ Alpheidae } & Alpheus macrocheles & Red pistol shrimp & $\mathrm{A}, \mathrm{P}$ \\
\hline & Alpheus glaber & Large pistol shrimp & $\mathrm{P}$ \\
\hline \multirow[t]{4}{*}{ Diogenidae } & Calcinus tubularis & Sedentary hermit crab & $\mathrm{A}, \mathrm{M}$ \\
\hline & Clibanarius erythropus* & Hermit crab & $\mathrm{A}, \mathrm{P}$ \\
\hline & Clibanarius aequabilis* & Hermit crab & $\mathrm{M}$ \\
\hline & Paguristes eremita & Hermit crab & $\mathrm{P}$ \\
\hline \multirow[t]{2}{*}{ Dromiidae } & Dromia marmorea & Marble sponge crab & A, M \\
\hline & Dromia personata & Linnaeus's sponge crab & $\mathrm{A}, \mathrm{P}$ \\
\hline Enoplometopodidae & Enoplometopus antillensis & Dwarf reef lobster & $\mathrm{M}$ \\
\hline Galatheidae & Galathea strigosa & Blue striped squat lobster & $\mathrm{A}, \mathrm{M}, \mathrm{P}$ \\
\hline \multirow[t]{5}{*}{ Hippolytidae } & Lysmata grabhami & Lady cleaner shrimp & M \\
\hline & Lysmata seticaudata & Monaco shrimp & $\mathrm{A}, \mathrm{M}, \mathrm{P}$ \\
\hline & Hippolyte inermis & Seagrass shrimp & $\mathrm{P}$ \\
\hline & Hippolyte varians & Variable seagrass shrimp & $\mathrm{A}, \mathrm{M}, \mathrm{P}$ \\
\hline & Thor amboinensis & Humpbacked shrimp & $\mathrm{M}$ \\
\hline \multirow[t]{2}{*}{ Palaemonidae } & Periclimenes sagittifer & Partner shrimp & $\mathrm{M}, \mathrm{P}$ \\
\hline & Tuleariocaris neglecta & Sea urchin shrimp & $\mathrm{M}$ \\
\hline Pandalidae & Plesionika narval & Unicorn shrimp & $\mathrm{A}, \mathrm{M}$ \\
\hline Rhynchocinetidae & Cinetorhynchus rigens & Atlantic dancing shrimp & A, M \\
\hline Stenopodidae & Stenopus spinosus & Mediterranean boxer shrimp & $\mathrm{A}, \mathrm{M}, \mathrm{P}$ \\
\hline \multirow{2}{*}{ Gnathophyllidae } & Gnathophyllum elegans & Spotted bumblebee shrimp & A, M \\
\hline & Gnathophyllum americanum & Striped bumblebee shrimp & A, M \\
\hline \multirow[t]{4}{*}{ Inachidae } & Inachus phalangium & Anemone spider crab & A, M, P \\
\hline & Inachus dorsettensis & Decorator spider crab & $\mathrm{P}$ \\
\hline & Macropodia rostrata & Spider crab & $\mathrm{P}$ \\
\hline & Stenorhynchus lanceolatus & Arrow crab & M \\
\hline \multirow{2}{*}{ Pisidae } & Pisa armata & Decorator crab & $\mathrm{A}, \mathrm{P}$ \\
\hline & Lissa chiragra & Decorator crab & $\mathrm{P}$ \\
\hline Paguridae & Pagurus prideaux & Prideaux's hermit crab & $\mathrm{M}, \mathrm{P}$ \\
\hline Plagusiidae & Percnon gibbesi* & Sally lightfoot & $\mathrm{A}, \mathrm{M}$ \\
\hline \multirow{2}{*}{ Xanthidae } & Platypodiella sp. & Gaudy clown crab & M \\
\hline & Euryozius bouvieri & Strawberry crab & $\mathrm{A}, \mathrm{M}$ \\
\hline \multicolumn{4}{|l|}{ Echinoderms } \\
\hline Antedonidae & Anthedon bifida & Atlantic feather star & M \\
\hline Arbaciidae & Arbaciella elegans & Elegant sea urchin & M \\
\hline Brissidae & Brissus unicolor & Big white heart urchin & M \\
\hline Cidaridae & Cidaris cidaris & Pencil urchin & $\mathrm{P}$ \\
\hline \multirow[t]{2}{*}{ Diadematidae } & Centrostephanus longispinus & Needle spined urchin & M \\
\hline & Diadema antillarum & Long spined sea urchin & $\mathrm{M}$ \\
\hline Echinasteridae & Echinaster sepositus & Spiny seastar & $\mathrm{M}, \mathrm{P}$ \\
\hline Loveniidae & Echinocardium cordatum & Small white heart urchin & $\mathrm{M}, \mathrm{P}$ \\
\hline Ophidiasteridae & Ophidiaster ophidianus & Purple seastar & $\mathrm{M}, \mathrm{P}$ \\
\hline Ophiodermatidae & Ophioderma longicauda* & Serpent star & $\mathrm{P}$ \\
\hline Spatangidae & Spatangus purpureus & Violet heart urchin & $\mathrm{P}$ \\
\hline
\end{tabular}

Portuguese waters that have a high commercial value, along with the growing restrictions on tropical marine ornamental collection and trade (Wood, 2001), seem to indicate that there is an increasing risk of these resources being heavily harvested. The lack of legislation regulating the capture of most species listed in the present work could be a major problem that could threaten the sustainable use of these marine resources.

To prevent the unsustainable use of these resources urgent regulating measures must be implemented that minimise the risk of jeopardising the conservation efforts of marine ecosystems currently being developed. To ensure that marine ornamental resources and their habitats are properly conserved and managed the following options (modified from Wood, 2001) should be evaluated:

- Banning the harvest and trade of all marine ornamental species present in Portuguese waters. Although tempting, this is always the most difficult approach to implement. One of the drawbacks associated with this approach may be the risk of involuntarily increasing the commercial value of species in high demand by reducing their supply. This measure can only be implemented if there is suitable surveillance of both collection areas and retail stores.

- Establishing sanctuary and no take zones. This is a method used for managing commercial marine 
TABLE 4. - Potential marine ornamental fish species from Portuguese waters (A, Azores; M, Madeira; P, Portugal mainland). * See text for explanation.

\begin{tabular}{|c|c|c|c|}
\hline Families & Species & Common name & Occurrence \\
\hline \multirow[t]{3}{*}{ Antennaridae } & Antennarius nummifer & Spotfin frogfish & $\mathrm{A}, \mathrm{M}$ \\
\hline & Antennarius senegalensis & Senegal frogfish & M \\
\hline & Histrio histrio & Sargassum frogfish & A \\
\hline Apogonidae & Apogon imberbis & Flame cardinal & $\mathrm{A}, \mathrm{M}, \mathrm{P}$ \\
\hline \multirow[t]{2}{*}{ Ballistidae } & Balistes punctatus & Spotted triggerfish & M \\
\hline & Balistes vetula & Queen trigerfish & A \\
\hline \multirow[t]{7}{*}{ Blennidae } & Coryphoblennius galerita* & Montagu's blenny & $\mathrm{A}, \mathrm{M}, \mathrm{P}$ \\
\hline & Lipophrys canevae & Reticulated blenny & $\mathrm{P}$ \\
\hline & Ophioblennius atlanticus & Redlip blenny & $\mathrm{A}, \mathrm{M}$ \\
\hline & Parablennius rouxi & Stripped blenny & $\mathrm{P}$ \\
\hline & Parablennius parvicornis* & Morocco blenny & $\mathrm{A}, \mathrm{M}$ \\
\hline & Parablennius rubber & Red blenny & A, M, P \\
\hline & Salaria pavo & Peacock blenny & $\mathrm{P}$ \\
\hline \multirow[t]{2}{*}{ Callionymidae } & Callionymus lyra & European dragonet & A, P \\
\hline & Callionymus pusillus & Festive-robe dragonet & $\mathrm{P}$ \\
\hline Carangidae & Selene dorsalis & Lookdown & $\mathrm{M}, \mathrm{P}$ \\
\hline Cottidae & Taurulus bubalis & Sea scorpion & $\mathrm{P}$ \\
\hline Dactylopteridae & Dactylopterus volitans & Flying gurnard & A, M, P \\
\hline \multirow{2}{*}{ Diodontidae } & Chilomyvterus atringa & Spiny puffer & M, P \\
\hline & Diodon hystrix & Porcupine fish & $\mathrm{A}, \mathrm{M}, \mathrm{P}$ \\
\hline \multirow[t]{3}{*}{ Gobiidae } & Gobius auratus & Golden goby & M, P \\
\hline & Gobius xanthocephalus & Red spotted goby & $\mathrm{M}, \mathrm{P}$ \\
\hline & Thorogobius ephippiatus & Leopard spotted goby & $\mathrm{M}, \mathrm{P}$ \\
\hline \multirow[t]{11}{*}{ Labridae } & Bodianus scrofa & Red hogfish & A, M \\
\hline & Centrolabrus truta & Atlantic wrasse & A, M \\
\hline & Coris julis & Rainbow wrasse & $\mathrm{A}, \mathrm{M}, \mathrm{P}$ \\
\hline & Ctenolabrus rupestris & Goldsinny wrasse & $\mathrm{P}$ \\
\hline & Xyrichthys novacula & Cleaver wrasse & A, M, P \\
\hline & Labrus bimaculatus & Cuckoo wrasse & $\mathrm{A}, \mathrm{M}, \mathrm{P}$ \\
\hline & Labrus bergylta & Ballan wrasse & $\mathrm{A}, \mathrm{M}, \mathrm{P}$ \\
\hline & Symphodus bailloni & Baillon's wrasse & $\mathrm{P}$ \\
\hline & Symphodus mediterraneus & Axillary wrasse & $\mathrm{M}, \mathrm{P}$ \\
\hline & Symphodus melops & Corkwing wrasse & A, P \\
\hline & Thalassoma pavo & Turkish wrasse & $\mathrm{A}, \mathrm{M}, \mathrm{P}$ \\
\hline Monacanthidae & Stephanolepis hispidus & Filefish & M \\
\hline \multirow[t]{3}{*}{ Muraenidae } & Enchelicore anatina & Fangtooth moray & $\mathrm{A}, \mathrm{M}$ \\
\hline & Gymnothorax polygonius & Spotted moray & M \\
\hline & Muraena augusti & Duke Augustus moray & $\mathrm{A}, \mathrm{M}$ \\
\hline Ostracidae & Acanthostracion notacanthus & Island boxfish & A \\
\hline \multirow[t]{3}{*}{ Pomacentridae } & Abudefduf luridus & Bluefin damsel & $\mathrm{A}, \mathrm{M}$ \\
\hline & Chromis chromis & Eastern-Atlantic damsel & $\mathrm{P}$ \\
\hline & Chromis limbata & Eastern-Atlantic damsel & $\mathrm{A}, \mathrm{M}$ \\
\hline \multirow{2}{*}{ Priacanthidae } & Heteropriacanthus cruentatus & Glasseye & M \\
\hline & Priacanthus arenatus & Atlantic bigeye & A, M \\
\hline \multirow[t]{5}{*}{ Serranidae } & Anthias anthias & Swallowtail sea perch & $\mathrm{A}, \mathrm{M}, \mathrm{P}$ \\
\hline & Callanthias ruber & Parrot sea perch & $\mathrm{A}, \mathrm{M}, \mathrm{P}$ \\
\hline & Mycteroperca fusca & Comb grouper & $\mathrm{A}, \mathrm{M}$ \\
\hline & Serranus cabrilla & Comber & $\mathrm{A}, \mathrm{M}, \mathrm{P}$ \\
\hline & Serranus scriba & Painted comber & $\mathrm{A}, \mathrm{M}, \mathrm{P}$ \\
\hline \multirow[t]{8}{*}{ Syngnathidae } & Enterlurus aequoreus & Snake pipefish & $\mathrm{P}, \mathrm{A}$ \\
\hline & Hipросатриs hipросатриs & Seahorse & $\mathrm{A}, \mathrm{M}, \mathrm{P}$ \\
\hline & Hipросатриs guttulatus & Seahorse & $\mathrm{P}$ \\
\hline & Nerophis lumbriciformis & Green pipefish & $\mathrm{P}$ \\
\hline & Nerophis ophidion & Green spotted pipefish & $\mathrm{P}$ \\
\hline & Syngnathus abaster & Blackstriped pipefish & $\mathrm{P}$ \\
\hline & Syngnathus acus & Great pipefish & A, P \\
\hline & Syngnathus typhle & Deepsnouted pipefish & $\mathrm{P}$ \\
\hline Scaridae & Sparisoma cretense & Parrotfish & $\mathrm{A}, \mathrm{M}, \mathrm{P}$ \\
\hline Scorpionidae & Scorpaena maderensis & Madeira scorpion fish & A, M \\
\hline \multirow[t]{4}{*}{ Tetraodontidae } & Canthigaster capistrata & Sharpnose puffer & M \\
\hline & Lagocephalus lagocephalus & Puffer fish & A, M, P \\
\hline & Sphoeroides marmoratus & Guinnean puffer & $\mathrm{A}, \mathrm{M}$ \\
\hline & Sphoeroides spengleri & Bandtail puffer & $\mathrm{A}, \mathrm{M}, \mathrm{P}$ \\
\hline
\end{tabular}

food-fisheries (see Frank and Brickman, 2001) that may also be applied to ornamental collection. Although such areas already exist in Portugal, enforcing the existing legislation is already a challenging task, particularly when dealing with illegal collection of specimens using scuba diving gear. 
TABLE 5. - Estimated retail value (in euros per specimen) and reason for vulnerability of some highly priced marine ornamental invertebrate organisms present in Portuguese waters. CV, Commercial Value; NAM, Novelty on the Aquarium Market; PIRLD, Present in the Intertidal Region or at Low Depths; UCM, Unsuitable Collection Method. * See text for explanation.

\begin{tabular}{|c|c|c|c|}
\hline Species & Common name & Commercial value (euros) & Reason for vulnerability \\
\hline Aplysina aerophoba & Golden sponge & $15-30$ & $\mathrm{CV}, \mathrm{UCM}$ \\
\hline Cerianthus membranaceus & Tube dwelling anemone & $20-40$ & $\mathrm{CV}, \mathrm{UCM}$ \\
\hline Telmatactis cricoides & Club tipped anemone & $30-50$ & $\mathrm{CV}, \mathrm{NAM}, \mathrm{UCM}$ \\
\hline Protula tubularia & Hard tube duster & $25-30$ & CV, UCM \\
\hline Sabella spallanzanii & Spiral feather duster & $8-12$ & CV, PIRLD \\
\hline Bolma rugosa & Star snail & $2-3 *$ & PIRLD \\
\hline Cerithium vulgatum & Common cerith & $1-2 *$ & PIRLD \\
\hline Hinia reticulata & Nassa snail & $1-3 *$ & PIRLD \\
\hline Cinetorhynchus rigens & Atlantic dancing shrimp & $15-20$ & NAM \\
\hline Clibanarius erythropus & Hermit crab & $5-6 *$ & CV, PIRLD \\
\hline Enoplometopus antillensis & Dwarf reef lobster & $25-40$ & $\mathrm{CV}, \mathrm{NAM}$ \\
\hline Lysmata grabhami & Lady cleaner shrimp & 30 & $\mathrm{CV}, \mathrm{NAM}$ \\
\hline Lysmata seticaudata & Monaco shrimp & 20 & CV, NAM, PIRLD \\
\hline Percnon gibbesi & Sally lightfoot & $6-8 *$ & CV, NAM, AIRLD \\
\hline Stenopus spinosus & Mediterranean boxer shrimp & $20-30$ & CV, NAM \\
\hline Diadema antillarum & Long spined sea urchin & 20 & CV, PIRLD \\
\hline Ophioderma longicauda & Serpent star & $10-12 *$ & CV, PIRLD \\
\hline
\end{tabular}

TABLE 6. - Estimated commercial value (in euros per specimen) and reason for vulnerability of some highly priced marine ornamental fish present in Portuguese waters. CV, Commercial Value; NAM, Novelty on the Aquarium Market; PIRLD, Present in the Intertidal Region or at Low Depths. * See text for explanation.

\begin{tabular}{|c|c|c|c|}
\hline Species & Common name & Commercial value (euros) & Reason for vulnerability \\
\hline Apogon imberbis & Flame cardinal & 25 & $\mathrm{CV}, \mathrm{NAM}$ \\
\hline Antennarius nummifer & Spotfin frogfish & $30-35$ & CV \\
\hline Histrio histrio & Sargassum frogfish & $20-35$ & $\mathrm{CV}$ \\
\hline Balistes punctatus & Spotted triggerfish & $30-60$ & $\mathrm{CV}$ \\
\hline Balistes vetula & Queen trigerfish & $30-60$ & CV \\
\hline Coryphoblennius galerita & Montagu's blenny & $15 *$ & PIRLD \\
\hline Selene dorsalis & Morocco blenny & $15^{*}$ & PIRLD \\
\hline Dactylopterus volitans & Lookdown & 300 & $\mathrm{CV}$ \\
\hline Diodon hystrix & Flying gurnard & 60 & $\mathrm{CV}$ \\
\hline Chilomycterus atringa & Porcupine fish & $25-45$ & $\mathrm{CV}$ \\
\hline Gobius auratus & Spiny puffer & $20-35$ & $\mathrm{CV}$ \\
\hline Bodianus scrofa & Golden goby & 20 & $\mathrm{CV}, \mathrm{NAM}$ \\
\hline Coris julis & Red hogfish & $20-50$ & $\mathrm{CV}, \mathrm{NAM}$ \\
\hline Thalassoma pavo & Rainbow wrasse & $15-30$ & $\mathrm{CV}, \mathrm{NAM}$ \\
\hline Stephanolepis hispidus & Turkish wrasse & $30-40$ & CV, NAM \\
\hline Enchelicore anatina & Filefish & $20-25$ & $\mathrm{CV}$ \\
\hline Muraena augusti & Fangtooth moray & $300-350$ & $\mathrm{CV}, \mathrm{NAM}$ \\
\hline Gymnothorax polygonius & Duke Augustus moray & $150-200$ & CV, NAM \\
\hline Acanthostracion notacanthus & Island boxfish & $15-30$ & $\mathrm{CV}$ \\
\hline Abudefduf luridus & Bluefin damsel & 25 & NAM \\
\hline Heteropriacanthus cruentatus & Glasseye & $40-50$ & $\mathrm{CV}$ \\
\hline Priacanthus arenatus & Atlantic bigeye & $50-65$ & $\mathrm{CV}$ \\
\hline Sparisoma cretense & Parrotfish & $40-50$ & $\mathrm{CV}, \mathrm{NAM}$ \\
\hline Scorpaena maderensis & Madeira scorpion fish & $15-20$ & $\mathrm{CV}$ \\
\hline Anthias anthias & Swallowtail sea perch & $30-50$ & $\mathrm{CV}, \mathrm{NAM}$ \\
\hline Hippocampus ramulosus & Seahorse & $25-30$ & CV, PIRLD \\
\hline Canthigaster rostrata & Sharpnose puffer & $20-35$ & CV, NAM \\
\hline
\end{tabular}

- Issuing collection permits. This measure would ensure that only certified, trained and conscientious collectors would be legally allowed to collect ornamental organisms for trading purposes. To enforce this measure retail stores would have to have a certificate provided by a licensed collector, which would ensure that the organisms for sale were not illegally collected. In addition, licensed collectors would almost certainly be the first ones to enforce this policy, preventing the action of illegal collectors.

- Creating certified wholesalers. This approach would considerably enhance accurate monitoring of ornamental species collection, since all licensed collectors would have to sell their products to a certified wholesaler. This procedure could play a vital role if 
species-specific quotas were established, since all captured organisms would be recorded. These certified wholesalers would be responsible for quarantining and maintaining collected animals before they were sold to retailers, ensuring that only healthy organisms were traded. Official wholesalers would also issue a certificate to retail stores confirming that the organisms for sale had been collected in a sustainable way. This measure could be implemented if hobbyists acted in a conscientious way by only buying certified ornamental organisms and reporting illegal trading to the proper authorities.

- Implementing the use of suitable gear and collecting methods. Although Portuguese law prohibits collecting marine life using scuba gear, for safety reasons licensed collectors should be allowed to operate using it. Therefore, ornamental organisms could be more easily selected and carefully collected. Nevertheless, each collector would be obliged to communicate to the proper authorities when and where they intended to collect the ornamental species. The only collecting gears allowed should be hand nets and small barrier nets (of a certain type and mesh size) for motile species and hand collection for attached or slow moving organisms. Using any kind of "anaesthetic" or destructive collecting method should not be allowed. This measure would not only promote lower post-harvest mortalities but also maximise profitability for collecting effort.

- Setting minimum and maximum size limits. Juvenile specimens, particularly fish, are popular in the aquarium trade. However, if there is relentless harvest pressure on wild juvenile specimens, as there has been in certain food fisheries (e.g. Myers and Quinn, 2002), stocks may be seriously threatened by a reduction in the number of organisms reaching sexual maturation size and a consequent decrease in recruitment may occur. This management measure would require a basic biological knowledge of the life cycle of target species, which unfortunately is still largely inexistent.

- Operating species-based quotas. This is a widely used practice in the food fishery industry as one of the key policies of sustainable marine resource management (e.g. Sutinen, 1999; Whiterell et al., 2000). Since some organisms are more eagerly collected than others, this measure would be highly beneficial. However, establishing a quota does not ensure that a proper conservation measure is being used. To establish appropriate quotas, considerable research effort is required concerning the life cycles and growth rates of target species, as well as the existence of feasible landing data like that used in food fisheries management (e.g. Koslow et al., 2000; Dunn, 2001). This practice, if properly monitored, can lead to a long lasting sustainable ornamental collection, even allowing an increase in the quotas initially established.

- Protection of rare, "key stone" species, and organisms with poor survivability in captivity. If research studies reveal that a certain species is becoming rare due to collection pressure, or plays a key role in the functioning of the ecosystem, the trade of this species should be banned. For example, some highly priced species present in Portuguese waters are known to play roles in cleaning symbioses (such as the wrasses from the genus Coris, Symphodus, and Thalassoma (Zander et al., 1999) and the shrimp from the genus Lysmata (Wirtz, 1995)). Although the actual significance of these associations is still not totally understood (see Spotte, 1998; Losey et al., 1999; Côté, 2000), the ecological impact of removing cleaners from the wild is unpredictable. In addition, species with very demanding captivity requirements make up $80 \%$ of post-harvest mortalities (Bunting et al., 2003), either in the wholesale/retail trade or the hobbyists' aquaria. Collecting and trading these species, such as seahorses, tube dwelling anemones, pen shells and feather stars should not be encouraged or should even be prohibited.

- Establishing closed seasons. Closure during the breeding season may prove to be a difficult task, since different species breed at different times of the year. However, such measures allow immature organisms to grow and reach maturity, maintaining the balance of wild populations. Again, such measures will only be effective if the biological studies needed to understand the life cycle of the potential target ornamental species are carried out.

- Culturing ornamental organisms. It has been suggested that this is the best approach for minimising the impacts of harvesting wild ornamental organisms, and may even be used to restore depleted ornamental populations (Ziemann, 2001). The current methodologies developed for the larviculture of marine organisms (Calado et al., 2003b; Holt, 2003) may be a precious contribution to the culture of ornamental species (Dhert et al., 1997). Although bottlenecks still impair the commercial culture of the majority of traded ornamental species (Ostrowski and Laidley, 2001), some ornamental 
shrimp species from Portuguese waters have already been successfully cultured in captivity on a commercial scale (Calado et al., 2003c). To promote the culture and trade of ornamental species raised in captivity, harvesting these organisms in the wild should be forbidden.

- Creating an ornamental research and management fund. A percentage ranging from 1 to $5 \%$ of the commercial value of each traded wild ornamental organism from Portuguese waters should be used to create an ornamental research and management fund. This fund could be used to enforce the application of future legislation, to finance research studies of the biology and culture of ornamental species and to implement monitoring programs to detect as early as possible any negative impact on marine ecosystems associated with harvesting ornamental species. Creating this kind of "eco-fee" would reassure conscientious hobbyists that they were contributing to the conservation and sustainable management of marine resources when buying legally collected organisms.

The suggestions presented here are only tentative guidelines for creating the legislation needed to regulate the collection of ornamental species. Although the present work deals specifically with organisms occurring in Portuguese waters it can be regarded as a case study.

In the near future, ornamental importing countries (other than the USA) may face marine resource management problems that were thought to be exclusive to ornamental exporting countries. The majority of European countries, particularly those in the Mediterranean basin, as well as northern and west African countries may have to rapidly "fill the gaps" in their legislation regarding marine ornamental collection and trade. The lack of legislation addressing this new problem will surely result in unsuitable exploitation of these highly priced organisms. However, if proper management measures are developed to ensure the sustainable harvest of marine ornamentals (see Bolker et al., 2002), an important alternative fishery may be created. Sustainable marine ornamental collection and trade may become an important income source, by creating new fisheries or adding value to several others by using discards or by-catches of existing food fisheries. By shifting the traditional food fisheries' target species to marine ornamentals, poor communities of fishers may be regenerated. If properly managed, these new fisheries may help to minimise the economic and social impacts to these communities caused by the crash of important food fisheries.

\section{ACKNOWLEDGEMENTS}

The author would like to thank Fundação para a Ciência e a Tecnologia (scholarship SFRH/BPD/ 18009/2004) of the Portuguese government for their financial support.

\section{REFERENCES}

Bolker, B.M., C.M. St. Mary, C.W. Osenberg, R.J. Schmitt and S.J. Holbrook. - 2002. Management at a different scale: marine ornamentals and local processes. Bull. Mar. Sci., 70: 733-748.

Bruckner, A.W. - 2001. Tracking the trade in ornamental coral reef organisms: the importance of CITES and its limitations. Aquarium Sci. Conserv. 3: 79-94.

Bryant, D., L. Burke, J. McManus and M. Spalding. - 1998. Reefs at risk. A map-based indicator of threats to the world's coral reefs. Washington DC: World Resource Institute.

Bunting, B.W., P. Holthus and S. Spalding. - 2003. The marine aquarium industry and reef conservation. In: J.C. Cato and C.L. Brown (eds.), Marine ornamental species - Collection, culture \& conservation, pp. 109-124. Iowa State Press, Iowa, USA.

Calado, R. Lin, J., Rhyne, A.L., Araújo, R., Narciso, L. - 2003a. Marine ornamental decapods - Popular, pricey and poorly studied. J. Crust. Biol., 23: 963-973.

Calado, R., J. Lin, A.L. Rhyne, R. Araújo and L. Narciso. - 2003b. Overview of marine ornamental shrimp aquaculture. In: J.C. Cato and C.L. Brown (eds.), Marine ornamental species Collection, culture \& conservation, pp. 221-230. Iowa State Press, Iowa, USA.

Calado, R., L. Narciso, S. Morais, A.L. Rhyne and J. Lin. - 2003c. A rearing system for the culture of ornamental decapod crustacean larvae. Aquaculture, 218: 329-339.

Chapman, F.A., S.A. Fitz-Coy, E.M. Thunberg and C.M. Adams. 1997. United States of America trade in ornamental fish. $J$. World Aquac. Soc., 28: 1-10.

Côté, I. M. - 2000. Evolution and ecology of cleaning symbioses in the sea. Oceanogr. Mar. Biol., 38: 311-355.

Daw, T.M., G.C.C. Rogers, P. Mapson and J.E. Kynoch. - 2001. Structure and management issues of the emerging ornamental fish trade in Eritrea. Aquarium Sci. Conserv., 3: 53-64.

Dhert, P., L.C. Lim, P. Candreva, H. Van Duffel and P. Sorgeloos. - 1997. Possible applications of modern fish larviculture technology to ornamental fish production. Aquarium Sci. Conserv., 1: $119-128$

Dunn, M.R. - 2001. The biology and exploitation of John dory, Zeus faber (Linnaeus, 1758) in the waters of England and Wales. ICES J. Mar. Sci., 58: 96-105.

Fosså, S.A. and A.J. Nielsen. - 2000. The modern coral reef aquarium. Volume 3. Birgit Schmettkamp Verlag, Bornheim.

Frank, K.T. and D. Brickman. - 2001. Contemporary management issues confronting fisheries science. J. Sea Res., 45: 173-187.

Friedlander, A.M. - 2001. Essential fish habitat and effective design of marine reserves: Application for marine ornamental fishes. Aquarium Sci. Conserv., 3: 135-150.

Holt, G.J. - 2003. Research on culturing early life stages of marine ornamental fish. In: J.C. Cato and C.L. Brown (eds.), Marine ornamental species - Collection, culture \& conservation, pp. 251-254. Iowa State Press, Iowa, USA

Koslow, J.A., G.W. Boehlert, J.D. Gordon, R.L. Haedrich, P. Lorance and N. Parin. - 2000. Continental slope and deep-sea fisheries: implications for a fragile ecosystem. ICES J. Mar. Sci., 57: 548-557.

Losey, G.S., A.S. Grutter, G. Rosenquist, J.L. Mahon and J.P. Zamzow. - 1999. Cleaning symbiosis: a review. In: V.C. 
Almada, R.F. Oliveira and E.J. Gonçalves (eds.), Behaviour and conservation of littoral fishes, pp. 379-395. Instituto Superior de Psicologia Aplicada, Lisboa, Portugal.

Michael, S.W. - 2001. A PocketExpert ${ }^{\mathrm{TM}}$ Guide - Marine fishes $500+$ essential to know aquarium species. T.F.H. Publications, Inc., New Jersey.

Myers, R.A. and T.J. Quinn II. - 2002. Estimating and testing nonadditivity in fishing mortality: implications for detecting a fisheries collapse. Can. J. Fish. Aquat. Sci., 59: 597-601.

Olivier, K. - 2003. World trade in ornamental species. In: J.C. Cato and C.L. Brown (eds.), Marine ornamental species Collection, culture \& conservation, pp. 49-63. Iowa State Press, Iowa, USA.

Ostrowski, A.C. and C.W. Laidley. - 2001. Application of marine foodfish techniques in marine ornamental aquaculture: Reproduction and larval first feeding. Aquarium Sci. Conserv., 3: 191-204.

Spotte, S. - 1998. "Cleaner" shrimps? Helgol. Meeresunters., 52: 59-64.

Sprung, J. - 2001. Oceanographic Series ${ }^{\mathrm{TM}}$ - Invertebrates a quick reference guide. Ricordea Publishing, Florida, USA.

Sutinen, J.G. - 1999. What works well and why: evidence from fishery-management experiences in OECD countries. ICES J. Mar. Sci., 56: 1051-1058.

Tlusty, M. - 2002. The benefits and risks of aquacultural production for the aquarium trade. Aquaculture, 205: 203-219.
Wabnitz, C., M. Taylor, E. Green and T. Razak. 2003. From ocean to aquarium. UNEP-WCMC, Cambridge, UK.

Wirtz, P. - 1995.Unterwasserführer Madeira, Kanaren, Azoren. Niedere Tiere. Delius Klasing, Naglschmid Verlag, Stuttgart, Germany.

Witherell, D., C. Pautzke and D. Fluharty. - 2000. An ecosystembased approach for Alaska groundfish fisheries. ICES J. Mar. Sci., 57: 771-777.

Wood, E. - 2001. Global advances in conservation and management of marine ornamental resources. Aquarium Sci. Conserv., 3: 65-77.

Wood, E.M. - 1985. Exploitation of coral reef fishes for the aquarium trade. Report to the Marine Conservation Society.

Zander, C.D., U. Meyer and A. Schmidt. - 1999. Cleaner fish symbiosis in European and Macaronesian waters. In: V.C. Almada, R.F. Oliveira R.F. and E.J. Gonçalves (eds.), Behaviour and conservation of littoral fishes, pp. 397-422. Instituto Superior de Psicologia Aplicada, Lisboa, Portugal.

Ziemann, D.A. - 2001. The potential for the restoration of marine ornamental fish populations through hatchery releases. Aquarium Sci. Conserv., 3: 107-117.

Scient. ed.: S. Zanuy

Received May 3, 2005. Accepted January 17, 2006.

Published online July 5, 2006. 